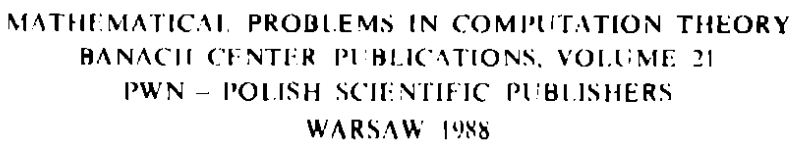

\title{
ОБ ОДНОМ ПОДХОДЕ К ОЦЕНКЕ ПРОСТРАНСТВЕННОЙ СЛОЖНОСТИ СХЕМ ИЗ ФУНКЦИОНАЛЬНЫХ ЭЛЕМЕНТОВ
}

\author{
С. А. ЛОЖКИН, А. И. РЫБКО, А. А. САПОЖЕНКО, \\ Ю. ХРОМКОВИЧ, Н. А. ШКАЛИКОВА
}

Московский государственный университет им. М. В. Ломоносова, Москва, СССР

\begin{abstract}
The problem of the layout of Boolean circuits into the plane is investigated. A new general approach for proving lower bounds on layout area is given. Using this approach, the strongest lower bound $\Omega\left(n^{2}\right)$ on the layout area of any Boolean circuit computing a specific Boolean function $g$ is obtained. Further, it is shown that the lower bound established is optimal, and that the three-dimensional layout of any Boolean circuit computing $g$ requires at least $n^{4 / 3}$ amount of space. To relate these results to combinational complexity the boolean circuit of the size $O\left(n \log _{2} n\right)$ computing $g$ is constructed.
\end{abstract}

\section{1. Введение}

Наиболее распространенной моделью вычисления дискретных функций являются схемы из функциональных элементов, сложность которых, понимаемая как число элементов, рассматривалась во многих работах (см., например, [5]). Однако, при реализации функций интегральными схемами существенным параметром оказывается площадь, которую занимает схема. При этом величина площади зависит не только от числа элементов, но и от структуры связей между ними. Как следует из работы [1], размешение на плоскости схемы, оптимальной по числу элементов, требует, как правило, такой площади, которая существенно больше оптимальной.

В работах [2], [4], [8] была рассмотрена математическая модель схем из функциональных элементов, размещенных на плоскости, в которой критерием сложности схемы служила занимаемая ею площадь. В работе [4] был установлен порядок, а в работе [2] - доказано существование асимптотики для сложности самой сложной функции от $n$ переменных. В работе [8] было показано, что сложность реализации всех элементарных конъюнкций от $n$ переменных равна по порядку $n \cdot 2^{n}$, сложность реализации всех булевых функций от $n$ переменных $-n \cdot 2^{2^{n}}$, 
сложность умножения двух $n$-разрядных двоичных чисел -- $n^{2}$, сложность реализации произвольной заданной перестановки $\boldsymbol{n}$ заданных булевских переменных -- $n^{2} \log n$. Что касается одной булевой функции $f:\{0,1\}^{n} \rightarrow$ $\rightarrow\{0,1\}$, то в работе [8] было доказано, что сложность реализации некоторых симметрических функций равна по порядку $n \log n$, а в работе [9] была построена специальная булевская функция, сложность которой равна по порядку $n^{3 / 2}$. Тем самым, для сложности плоской реализации конкретных булевских функций были получены нелинейные нижние оценки.

Используя технику получения нижних оценок из [8], мы введем понятие коммуникативной сложности схем из функциональных элементов, с помощью которого сформулируем общий подход к доказательству нижних оценок сложности пјоской реализации булевских функций. Применяя этот подход, получим квадратичную нижнюю оценку для сложности плоской реализации одной специальной булевской функции. С другой стороны, мы докажем, что эта оценка является точной по порядку. Будег доказана также нижняя оценка порядка $n^{4 / 3}$ для сложности объемной реализации этой функции и верхняя оценка порядка $n \log n$ для числа функциональных элементов.

\section{2. Основные определения}

Введем понятие коммуникативной сложности для схем из функциональных элементов. Отметим, что это понятие суцественно отличается от понятия коммуникативной сложности из [3].

Пусть $F$ - система булевских функций вида $\{0,1\}^{N} \rightarrow\{0,1\}^{m}$ ог $N$ переменных $X=\left\{x_{1}, x_{2}, \ldots, x_{N}\right\}$ и пусть $Y \subseteq X$. Рассмотрим произвольную схему $S$ из функциональных элементов в каком-либо конечном базисе, которая реализует $F$. Пусть $\Pi_{Y, S}-$ множество всех разбиений $\pi_{Y}$ вершин схемы $S$ на два непересекающихся подмножества, каждое из которых содержит не менее [|Y|/2] переменных из $Y$. Обозначим через $c\left(\pi_{\gamma}\right)$ число ребер, соединяющих вершины из различных подмножеств разбиения $\pi_{Y}$. Положим:

$$
\begin{gathered}
c_{Y}(S)=\min \left\{c\left(\pi_{Y}\right) \mid \pi_{Y} \in \Pi_{Y, S}\right\}, \\
c_{Y}(F)=\min \left\{c_{Y}(S) \mid S \text { реализует } F\right\}, \\
c(F)=\max \left\{c_{Y}(F)|Y \subseteq X,| Y|\geqslant| X \mid / 3\right\} .
\end{gathered}
$$

Величину $c(F)$ будем называть коммуникативной сложностью системы функций $F$. Легко видеть [3], что для любой системы булевских функций $F$ от $N$ переменных $c(F) \leqslant N / 2$.

Формальное определение математической модели схем из функцио- 
нальных элементов, размецценных на плоскости, можно найти в [2], [4], [8]. Это определение основывается на понятии схемы из клеточных элементов, которая с содержательной точки зрения представляет собой плоскую прямоугольную решетку, в каждой клетке которой расположен либо функциональный, либо коммутационный элемент. На каждой стороне клетки расположено не более одного входа или выхода соответствующего элемента, с помощью которого этот элемент соединяется с соседним элементом. Входы и выходы всей схемы расположены (вообще говоря произвольно) по ее границе. Под сложностью схемы понимается число клеток прямоугольника, который она занимает, а под сложностью $A(F)$ системы булевских функций $F$ - минимальная из площадей (сложностей) схем, ее реализующих.

\section{3. Подход к получению нижних оценок}

Основой предлагаемого подхода является следующая

ТЕОРЕмА. Пусть $F-$ система булевских функций вида $\{0,1\}^{N} \rightarrow$ $\rightarrow\{0,1\}^{m}$. Тогдa

$$
A(F) \geqslant \frac{1}{12}(N+m) c(F) .
$$

Доказательство. Пусть $K$ - схема из клеточных элементов размеров $a$ на $b$, реализующая систему $F$. Пусть $a \geqslant b$ и, следовательно, $a \geqslant(m+N) / 4$. Возьмем произвольное подмножество $Y$ множества переменных $X=\left\{x_{1}, \ldots, x_{n}\right\}$ системы $F$ такое, что $|Y| \geqslant N / 3$. Рассмотрим два случая в зависимости от того, существует или нет прямая $l$ параллельная стороне длины $b$ схемы $K$, которая разделяет входные переменные $Y$ на две части, содержащие не менее чем [|Y|/2] переменных. Если такой прямой не существует, то по крайней мере на одной из сторон длины $b$ схемы $K$ должно быть расположено не менее половины входов из $Y$. Следовательно,

$$
b \geqslant N / 6, \quad a b \geqslant \frac{m+N}{4} \cdot \frac{N}{6}
$$

и, так как $c_{Y}(F) \leqslant c(F) \leqslant N / 2$, то

$$
a b \geqslant \frac{1}{12}(m+N) c_{Y}(F) .
$$

Если такая прямая существует, она порождает некоторое разбиение $\pi_{Y}$ обынной схемы из функциональных элементов $S$, соответствующей схеме $K$. Тем самым,

$$
b \geqslant c\left(\pi_{Y}\right) \geqslant c_{Y}(F) \quad \text { и } \quad a b \geqslant \frac{m+N}{4} c_{Y}(F) .
$$


В любом из этих случаев для каждого $Y \subseteq X$ такого, что $|Y| \geqslant N / 3$ получено неравенство

$$
a b \geqslant \frac{m+N}{12} c_{Y}(F)
$$

которое доказывает теорему.

Введенное выше понятие коммуникативной сложности булевских функций имеет общие черты с понятием коммуникативной сложности VLSI-схем, предложенной в [7]. Коммуникативная сложность схем из функциональных элементов, реализующих $F$, позволяет получать нижние оценки для $A(F)$, в то время как коммуникативная сложность VLSI-схем помогает получать нижние оценки только для пространственно-временного параметра $A T^{2}$. Это связано с тем, что соответствующие модели вычисления имеют следующие основные отличия:

1. все процессоры в VLSI-схеме работают синхронно в каждый из дискретных моментов времени $t=0,1, \ldots$ пока продолжается вычисление, тогда как каждый клеточный элемент срабатывает в течение вычисления только один раз;

2. в схеме из клеточных элементов каждая входная переменная имеет свой вход, в то время, как в VLSI-схеме через один и тот же вход в разные моменты времени могут поступать разные переменные.

Обозначим через $\mathrm{COMM}(f)$ коммуникативную сложность булевской функции $f$ в VLSI-модели [6]. Легко показать, что

$$
\operatorname{COMM}(f) \leqslant c(f)
$$

Так как для почти всех булевских функций от $N$ переменных $\operatorname{COMM}(f) \geqslant[N / 2]$ (см. [6]), то для почти всех функций от $N$ переменных будет иметь место равенство $c(f)=[N / 2]$.

\section{4. Квадратичная оценка площади для одной булевской функции}

Получим квадратичную нижнюю и квадратичную верхнюю оценки величины $A\left(g_{n}\right)$ для булевской функции $g_{n}$ от переменных $\tilde{x}=\left(x_{1}, x_{2}, \ldots, x_{2 n}\right)$, $\tilde{y}=\left(y_{1}, y_{2}, \ldots, y_{2 n}\right), \tilde{u}=\left(u_{1}, \ldots, u_{2 n}\right), \tilde{v}=\left(v_{1}, \ldots, v_{2 n}\right)$ определенной следующим образом:

$$
g_{n}(\tilde{u}, \tilde{v}, \tilde{x}, \tilde{y})=\sum_{i=1}^{s}\left(x_{h_{k}(\tilde{u})} \sim y_{h_{k}(\tilde{v})}\right)
$$

где

$$
s=s(\tilde{u}, \tilde{v})=\min \left\{\sum_{i=1}^{2 n} u_{i}, \sum_{j=1}^{2 n} v_{j}\right\}
$$


a $h_{k}(\tilde{z})$-- номер той координаты набора значений переменных $\tilde{z}$, в которой находится $k$-я (считая слева направо) единица. Функция $g_{n}$ равна 1 тогда и только тогда, когда поднаборы наборов значений переменных $\tilde{x}$ и $\tilde{y}$, выделяемые единицами наборов значений переменных $\tilde{u}$ и $\tilde{v}$ соответственно, совпадают в первых $s(\tilde{u}, \tilde{v})$ координатах.

ЛЕМмА 1. $c\left(g_{n}\right) \geqslant n$.

Доказательство. Обозначим через $W$ множество всех переменных функции $g_{n}$. Рассмотрим подмножество $Z$ множества $W$, состоящее из переменных наборов $\tilde{x}$ и $\tilde{y}$. Нам достаточно доказать, что $c_{z}\left(g_{n}\right) \geqslant n$.

Предположим, что это не так, то есть, существует схема из функциональных элементов $S$, реализующая $g_{n}$, и ее разбиение $\pi_{z}$, для которого $c\left(\pi_{z}\right) \leqslant n-1$. Пусть $\tilde{q}=\left(q_{1}, \ldots, q_{r}\right)$ - набор тех вершин схемы $S$, которые соответствуют выходам элементов или входам $S$, переходящим из одной части разбиения $\pi_{Z}$ в другую. Очевидно, что $r \leqslant c\left(\pi_{z}\right) \leqslant(n-1)$. Для каждого набора $\tilde{\delta}$ значений входных переменных обозначим через $\tilde{\eta}(\tilde{\delta})$ набор значений булевских функций, реализуемых в вершинах $q_{1}, q_{2}, \ldots, q_{r}$.

Пусть в одной части разбиения $\pi_{z}$ оказалось $p$ переменных из набора $\tilde{x}$ и $m$ переменных из набора $\tilde{y}$, где $p+m=2 n$. Без ограничения общности можно считать, что $m \geqslant p$ и поэтому $m \geqslant n$, и что в первой части разбиения $\pi_{z}$ находятся переменные $X^{\prime}=\left\{x_{i_{1}}, \ldots, x_{i_{n}}\right\}$, а во второй части -- переменные $Y^{\prime}=\left\{y_{j_{1}}, \ldots, y_{j_{n}}\right\}$. Выберем значения $\tilde{\alpha}$ и $\tilde{\beta}$ переменных й и $\tilde{v}$ так чтобы, для всех $k=1,2, \ldots, n, h_{k}(\tilde{\alpha})=i_{k}, h_{k}(\widetilde{\beta})=j_{k}$, а число 1 в каждом из наборов $\tilde{\alpha}$ и $\tilde{\beta}$ было равно $n$.

Рассмотрим множество $M$ всех таких наборов $\tilde{\delta}$ значений переменных из $W$, для которых $\tilde{u}=\tilde{\alpha}, \tilde{v}=\tilde{\beta}$, переменные $x_{i}$ и $y_{j}$, не входящие, соответственно, в $X^{\prime}$ и $Y^{\prime}$, равны 1 , и, кроме того, $g_{n}(\tilde{\delta})=1$. Очевидно, что $|M|=2^{n}$. Так как $r \leqslant c\left(\pi_{z}\right) \leqslant(n-1)$, то найдутся два различных набора $\tilde{\delta}^{\prime}$ и $\tilde{\delta}^{\prime \prime}$ из $M$, для которых $\tilde{\eta}\left(\widetilde{\delta^{\prime}}\right)=\tilde{\eta}\left(\widetilde{\delta^{\prime \prime}}\right)$. Обозначим через $\tilde{\gamma}^{\prime}$ набор, который получается заменой в наборе $\widetilde{\delta}^{\prime}$ координат, связанных с множеством переменных $X^{\prime}$, на соответствующие координаты набора $\widetilde{\delta^{\prime \prime}}$, а через $\tilde{\gamma}^{\prime \prime}$ набор, который получается заменой в наборе $\widetilde{\delta}^{\prime \prime}$ координат, связанных с множеством переменных $Y^{\prime}$, на соответствующие координаты набора $\tilde{\delta}^{\prime}$. Очевидно, что $g_{n}\left(\tilde{\gamma}^{\prime}\right)=g_{n}\left(\tilde{\gamma}^{\prime}\right)=0$.

Предположим, что выход схемы находится в первой части разбиения $\pi_{z}$ и придем к противоречию, показав, что на наборе $\tilde{\gamma}^{\prime \prime}$ на выходе схемы должна появиться 1. Рассмотрим первую часть схемы $S$ как самостоятельную схему $S_{1}$, входами которой являются те входы $S$, которые в нее попали, и часть вершин $q_{1}, q_{2}, \ldots, q_{r}$, а выходом - выход схемы $S$. На наборах $\tilde{\delta}^{\prime \prime}$ и $\tilde{\gamma}^{\prime}$ значения всех входов схемы $S_{1}$ одинаковы и, следовательно, на наборе $\tilde{\gamma}^{\prime \prime}$ на выходе схемы $S_{1}$, а значит и на выходе $S$ должна пояавиться 1. Если выход схемы $S$ расположен во второй половине 
разбиения $\pi_{z}$, то аналогичные рассуждения будут справедливы для наборов $\tilde{\gamma}$ и $\tilde{\delta}^{\prime}$. Полученное противоречие доказывает лемму.

СледСтвиЕ. $A\left(g_{n}\right) \geqslant \frac{3}{4} n^{2}$.

Доказательство следует из теоремы и леммы 1.

Лемма 2. $A\left(g_{n}\right) \leqslant d n^{2}$, где $d-$ некоторая константа, зависячая от базиса.

Доказательство. Построим такую схему $K$ из клеточных элементов, которая реализует функцию $g_{n}$ и имеет площадь, равную по порядку $n^{2}$. Схема $K$ включает в себя прямоутольную решетку, составленную из одинаковых по размерам, ориентации и функционированию прямоугольных блоков (схем) $K_{i, j}, i, j=1,2, \ldots, 2 n$. Каждый такой блок имеет 7 входов и 7 выходов, пронумерованных числами $1,2, \ldots, 7$ (см. рис. 1). Входы и выходы блоков, имеющие өдин и тот же номер, будем называть соответствующими. Функционирование одного блока $K_{i, j}$ может быть описано следующим образом: значения на выходах 1, 2, 6, 7 всегда равны значениям на соответствущих входах; значения на выходах 3-5 могут отличаться от значений соответствующих входов только тогда, на всех входах 2-6 имеются единичные значения; в этом случае значения на выходах 3, 4 равны нулю, а на выходе 5 реализуется функция совпадения входов 1 и 7, то есть функция $x_{i} \sim y_{j}$.

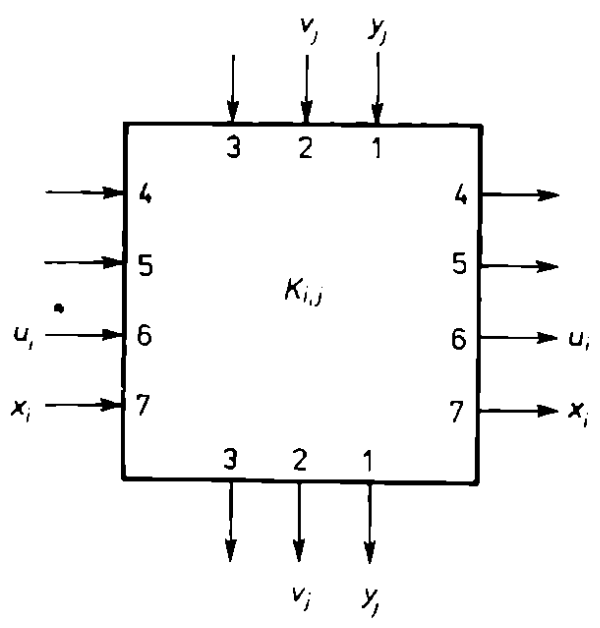

Pис. I

В схеме $K$ на входы 1,2 и 3 каждого блока $K_{i, j}$ при $i>1$ подаются соответствующие выходы блока $K_{i-1, j}$, а при $i=1$ - переменные $y_{j}, v_{j}$ и константа 1; аналогично организовано соединение входов и выходов соседних блоков $K_{i, j-1}, K_{i, j}, i, j=1, \ldots, 2 n$, причем на входы 4,5 блока $K_{i, 1}$ подается константа 1 , а на входы 6, 7 этого блока - переменные $u_{i}, x_{i}$ (см. рис. 2). Выход 5 каждого блока $K_{i, 2 n}, i=1, \ldots, 2 n$, подается на 1-й вход блока $D_{i}$, реализующего конъюнкцию. На 2-й вход блока $D_{i}$ при 


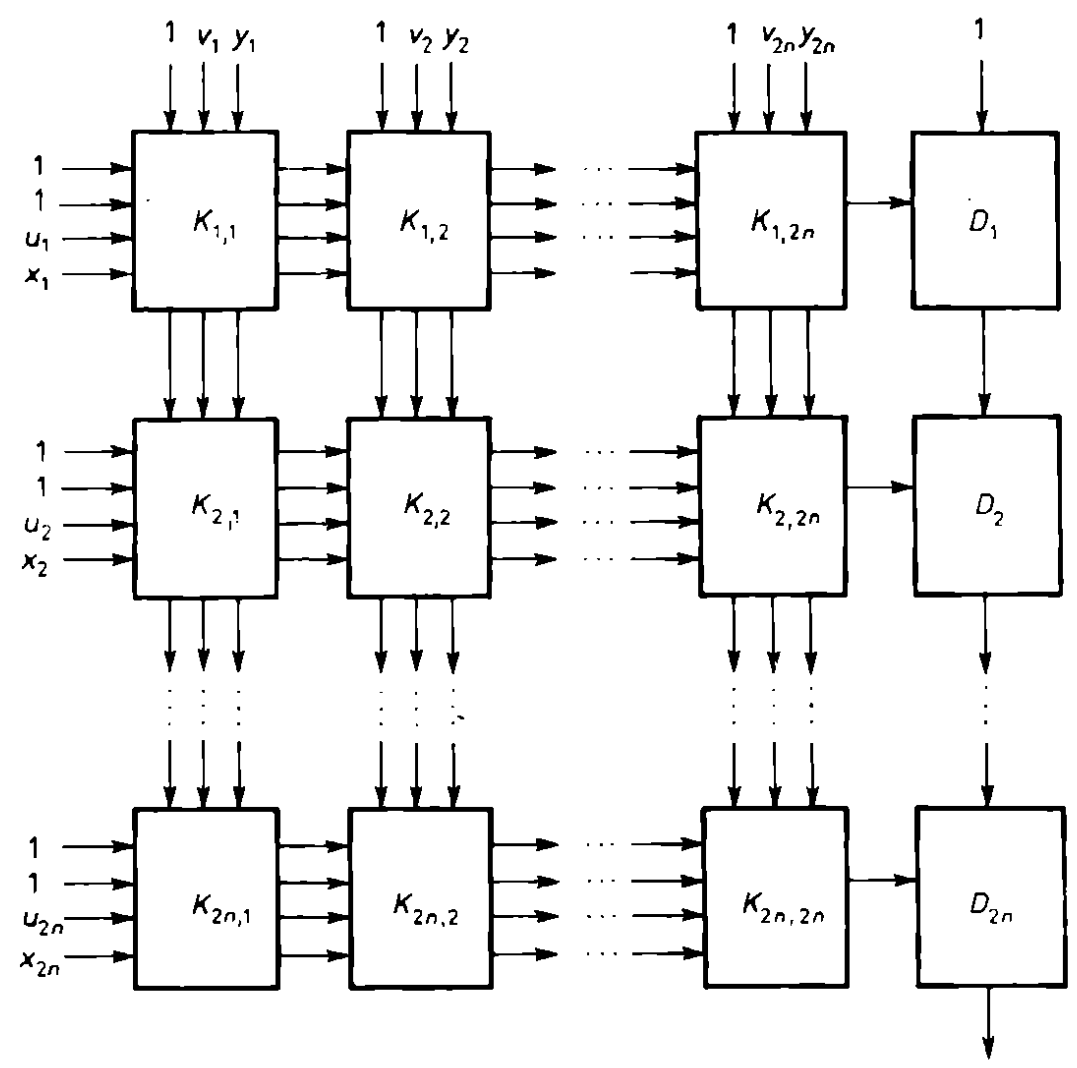

Рис. 2

$i>1$ подается выход блока $D_{i-1}$, а при $i=1$ - константа 1. Выходом схемы является выход блока $D_{2 n}$.

Нетрудно убедиться в том, что при любом наборе значений переменных $\tilde{u}$ и $\tilde{v}$ все блоки $K_{i, j}, i, j=1, \ldots, 2 n$, для которых сомножитель $\left(x_{i} \sim y_{j}\right)$ не входит в конъюнкцию (1), работают как коммутационные элементы, передающие значения входов на соответствующие выходы. Сомножители конъюнкции (1), реализованные при этом на выходах 5 остальных блоков $K_{i, j}$, проходят на входы блоков $D_{i}$ и на выходе схемы возникает значение функции $g_{n}$.

Очевидно, что каждый из описанных выше блоков может быть реализован с константной сложностью в произвольном полном базисе из функциональных и коммутационных элементов, и поэтому для некоторой константы $d$, зависящей от базиса,

$$
A\left(g_{n}\right) \leqslant d n^{2} .
$$

Лемма доказана.

В работе [9] рассматривалось размещение схем из функционалыных элементов не только в двумерном, но и в трехмерном пространстве, причем мерой сложности трехмерной схемы служил занимаемый ею объем. Там было доказано, что для каждой трехмерной схемы объема $V$ можно построить эквивалентную ей плоскую схему, плошадь которой по 
порядку не больше чем $V^{3 / 2}$. В силу доказанных выше теоремы и леммы 1 , отсюда следует, что сложность объемной реализации функции $g_{n}$ по порядку не меньше чем $n^{4 / 3}$.

В дальнейшем через $L(F)$ будем обозначать минимальное число функциональных элементов из какого-либо конечного полного базиса, достаточное для построения схемы, реализующей систему булевских функций $F$. Для булевского набора $\tilde{\sigma}=\tilde{\sigma}^{r}=\left(\sigma_{1}, \ldots, \sigma_{r}\right)$ введем также обозначения: $\|\tilde{\sigma}\|=\sum_{i=1}^{r} \sigma_{i}$ и $|\tilde{\sigma}|=\sum_{i=1}^{r} \sigma_{i} 2^{i-1}$.

ЛеммА 3. Для некоторой константы $d_{1}$, зависящей от базиса, справедливо неравенство $L\left(g_{n}\right) \leqslant d_{1} n \log n$.

Доказательство. Для $q=2^{p-1}, p=2,3, \ldots$ рассмотрим систему булевских функций $\boldsymbol{R}_{q}$ вида $\{0,1\}^{2 q+p} \rightarrow\{0,1\}^{2 q}$, которая переводит набор значений входных переменных $\tilde{x}^{q}, \tilde{u}^{q}, \tilde{s}^{p}$ в набор значений выходных переменных $\tilde{w}^{2 q}$ так, что при $\tilde{u}=\tilde{o}^{q} \tilde{w}=\tilde{o}^{2 q}$, а при $\tilde{u} \neq \tilde{\sigma}^{q}$ для всех $k=1,2, \ldots,\|\tilde{u}\|, w_{|s|+k}=x_{h_{k}(\tilde{\hat{u}})}$, тогда как все остальные переменные $w_{j}$ равны 0. Покажем, что

$$
L\left(R_{2 q}\right) \leqslant 2 L\left(R_{q}\right)+d_{2} q,
$$

где $d_{2}$ - некоторая константа, зависящая от базиса. Схема, которая реализует $R_{2 q}$, показана на рис. 3 , где $\tilde{x}^{\prime}, \tilde{x}^{\prime \prime}, \tilde{u}^{\prime}, \tilde{u}^{\prime \prime}, \tilde{s}$ и $\tilde{s}^{\prime}-$ наборы переменных $\left(x_{1}, \ldots, x_{q}\right),\left(x_{q+1}, \ldots, x_{2 q}\right),\left(u_{1}, \ldots, u_{q}\right),\left(u_{q+1}, \ldots, u_{2 q}\right)$, $\left(s_{1}, \ldots, s_{p-1}\right)$ и $\left(s_{p}, s_{p+1}\right)$ соответственно. Блок $M$ вычисляет набор $\tilde{\tau}=\left(\tau_{1}, \ldots, \tau_{p+1}\right)$, для которого $|\tilde{\tau}|=\left\|\tilde{u}^{\prime}\right\|$. Блок $\Sigma$ вычисляет набор $\tilde{\sigma}=\left(\tilde{\sigma}^{\prime}, \tilde{\sigma}^{\prime \prime}\right)$, где $\tilde{\sigma}^{\prime}=\left(\sigma_{1}, \ldots, \sigma_{p-1}\right), \tilde{\sigma}^{\prime \prime}=\left(\sigma_{p}, \sigma_{p+1}, \sigma_{p+2}\right)$, для которого

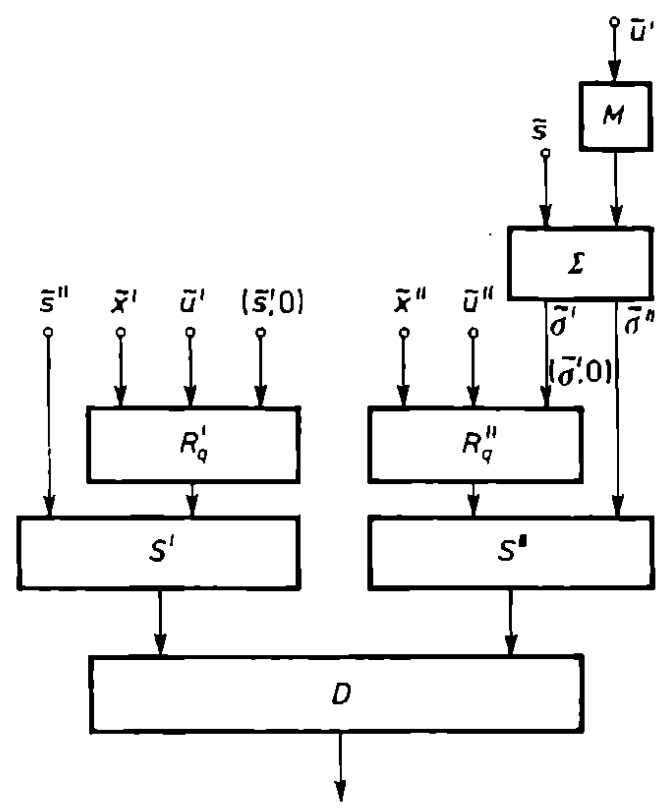

Puc. 3 
$|\tilde{\sigma}|=|\tilde{s}|+|\tilde{\tau}|$, а блоки $R_{q}^{\prime}$ и $R_{q}^{\prime \prime}$ реализуют системы функций $R_{q}$ от переменных $\left(\tilde{x}^{\prime}, \tilde{u}^{\prime},(\tilde{s}, 0)\right)$ и $\left(\tilde{x}^{\prime \prime}, \tilde{u}^{\prime \prime},\left(\tilde{\sigma}^{\prime}, 0\right)\right)$ соответственно. Блок $S^{\prime}$ (соответственно $S^{\prime \prime}$ ) имеет $4 q$ выходов и сдвигает выходы блока $R_{q}^{\prime}$ (соответственно $R_{q}^{\prime \prime}$ ) на $q\left|\tilde{s}^{\prime}\right|$ (соответственно $q\left|\tilde{\sigma}^{\prime \prime}\right|$ ), разрядов вправо, присваивая нулевые значения ,свободным” выходным переменным. Блок $D$ выполняет поразрядную дизъюнкцию выходов блоков $S^{\prime}$ и $S^{\prime \prime}$. Неравенство (2) следует из того, что блоки $M, \Sigma, S^{\prime}, S^{\prime \prime}$ и $D$ можно реализовать с линейной относительно числа их входов сложностью. С помощью (2) легко показать, что для некоторой константы $d_{3}$, зависящей от базиса, и любого $p=1,2, \ldots$

$$
L\left(R_{2 p}\right) \leqslant d_{3} p \cdot 2^{p} .
$$

Схему, которая реализует функцию $g_{n}$ и имеет сложность, удовлетворяющую требованиям леммы, можно построить из двух схем $R_{q}$, где $q=2^{p-1}$, а $\left.p=\right] \log _{2} 2 n[+1$, и схемы сравнения двух q-разрядных наборов. На входы первой схемы $R_{q}$ подаются наборы $\left(\tilde{x}^{2 n}, \tilde{O}^{q-2 n}\right),\left(\tilde{u}^{2 n}, \tilde{O}^{q-2 n}\right)$ и набор $\tilde{\alpha}$, для которого $|\tilde{\alpha}|=q-\min \{\|\tilde{u}\|,\|\tilde{v}\|\}$, а на входІ: второй схемы $\boldsymbol{R}_{q}$ - наборы $\left(\tilde{y}^{2 n}, \tilde{O}^{q-2 n}\right),\left(\tilde{v}^{2 n}, \tilde{O}^{q-2 n}\right)$ и $\tilde{\alpha}$. Схема срявнения сравнивает первые $q$ выходов указанных схем $R_{q}$. Так как сложность схемы, вычисляющей набор $\bar{\alpha}$, и сложность схемы сравнения линейны относительно $q$, сложность всей схемы, реализующей функцию $g_{n}$, определяется сложностью схем $R_{q}$ и имеет порядок $n \log n$.

Результаты данной статьи были получены при обсуждении проблемы на одном из семинаров в международном математическом центре им. С. Банаха.

\section{Литература}

[1] A. Albrecht, Komplexitatstheoretische Aspecte des Antwurfs von VLSI-Systemen, Prepr. Humboldt-Univ. Berlin, Sect. Math. 28 (1982), 36.

[2] -, О схемах из клеточных элементов, Проблемы кибернетики, вып. 33, М., Наука (1978), 209-214.

[3] J. Hromkovic, Linear lower bounds on unbounded fan-in Boolean circuits, Information Processing Letters 21 (2), 71-75.

[4] С. С. Кравцов, О реализачии фуикций алгебры логики в одном классе схем из функииональных и коммутационных элементов, Проблемы кибернетики, вып. 19, М., Наука (1967), 274-284.

[5] О. Б. Лупанов, O синтезе некоторых классов управляющих систем, Проблемы кибернетики, вып. 10, М., Физматгиз 1963, 63-97.

[6] C. H. Papadimitriou, M. Sipser, Communication complexity, J. of Computer and System Sciences 28 (2) (1984), 261-269.

[7] C. D. Thompson, A complexity theory for VLSI, Ph. D. dissertation, Dept. Comput. Sci., Carnegie-Mellon Univ., Pittsburgh, PA, August 1980.

[8] Н. А. Ш ка ли ков В, О сложности реализачии некоторых функиий клеточными схемами, Сборник работ по математической кибернетике, вып. І, М., ВЦ АН СССР (1976), $102-115$. 
512

С. А. ПОЖКИН, А. И. РЫБКО, А. А. САПОЖЕНКО, Ю. ХРОМКОВИЧ, Н. А. ШКАЛИКОНА

[9] -, О соотношении сложностей плоских и объемных схем из функциональных элементов, Методы дискретного анализа в оценках сложности управляющих снстем, вып. 38 , Новосибирск (1982), 87107.

Presented to the semester Mathematical Problems in Computation Theory

September 16-December 14, 1985 